\title{
Polymer-supported reagents and catalysts: increasingly important tools for organic synthesis
}

The use of polymer supports in organic synthesis began with solid-phase synthesis where the synthetic target is synthesized attached to the polymer. For a variety of reasons, including ease of reaction monitoring and product characterization, shorter method development time, etc., the role of the polymer has gradually shifted to supporting reagents for reacting with solution-phase substrates in what is sometimes referred to as polymer-assisted synthesis. Nowadays such use of polymer-supported reagents is as common, if not more so, than solid-phase synthesis, and a great number of such reagents have been reported in the literature and are commercially available. However, despite intensive research efforts over the past few years, the number of polymer-supported reagents known is still just a small fraction of the reagents commonly used in traditional solution-phase organic synthesis. Therefore, as the desire for faster production of compound collections with greater structural complexity increases, the need for polymer-supported reagents capable of effecting different reactions and those with greater efficiency grows as well. This Symposium-in-Print highlights some of the recent progress in developing new polymer-supported reagents and showcases some of the different strategies for enhancing their performance and improving methods for their preparation.

The cover picture displays an extremely rare English "ladder" scale by Degrave, Short, Fanner and Company, circa mid-19 ${ }^{\text {th }}$ century (from the collection of K.D. Janda). The scale is made up of three beams that can measure materials in units from 0.5 to 9 ounces. Upon each "ladder step" are molecules that depict applications of polymer supports in organic synthesis. Each of these applications has specific requirements of the polymer regarding solvent compatibility, reactivity, porosity, etc. Thus, one polymer does not fit all applications, just as there is no universal solvent, and striking the proper balance between polymer structure and synthetic use is essential. To the lower left of picture are listed some of the polymer supports used in the research described in this issue. Judging by the number and variety of materials listed, it is quite clear that the research regarding polymer-supported reagents and catalysts the polymers used to support them is an active and dynamic field and that many important discoveries are still to be made.

Patrick H. Toy Department of Chemistry The University of Hong Kong

Pokfulam Road

Hong Kong, People’s Republic of China

phtoy@hku.hk

Min Shi

School of Chemistry \& Pharmaceutics East China University of Science and Technology 130 Meilong Road Shanghai 200237, People's Republic of China mshi@pub.sioc.ac.cn 